Les ANNALES Les Annales de droit

DE DROIT

12 | 2018

Varia

\title{
Les nouveaux mouvements constitutionnels dans le monde arabe (2011-2016)
}

New constitutional changes in the Arab world (2011-2016)

\section{Malik Boumédiene}

\section{(2) OpenEdition}

\section{Journals}

Édition électronique

URL : http://journals.openedition.org/add/1006

DOI : $10.4000 /$ add. 1006

ISSN : 2606-1988

Éditeur

Presses universitaires de Rouen et du Havre

Édition imprimée

Pagination : 87-122

ISBN : 979-10-240-0937-7

ISSN : 1955-0855

\section{Référence électronique}

Malik Boumédiene, «Les nouveaux mouvements constitutionnels dans le monde arabe (2011-2016) », Les Annales de droit [En ligne], 12 | 2018, mis en ligne le 05 février 2019, consulté le 05 décembre 2019. URL : http://journals.openedition.org/add/1006 ; DOI : 10.4000/add.1006 


\title{
Les nouveaux mouvements constitutionnels dans le monde arabe (2011-2016)
}

\author{
Malik BOUMÉDIENE
}

Le monde arabe fait l'objet depuis plus de six ans de toutes les attentions et se trouve sous les projecteurs de l'actualité, faisant ainsi l'objet de diverses études ${ }^{1}$. Cependant, ces études concernant les " révolutions " arabes sont, avant tout, politiques ${ }^{2}$. Les études juridiques restent, le plus souvent, circonscrites à une question spécifique ou encore à un

1. Voir Baudouin Dupret, "Maroc, réformer sans bouleverser», dans Afrique $d u$ Nord, Moyen-Orient: printemps arabes, trajectoires variées, incertitudes persistantes, Paris, La Documentation française, 2012-2013, p. 147; Alaya Allani, «Transition démocratique en Tunisie et rôle de la collaboration internationale", dans Où va le monde arabe?, Paris, Bonnier, 2012, p. 55; Flavien Bourrat, «Maroc, Tunisie et Algérie: les évolutions politiques depuis le début des révolutions arabes », dans Afrique du Nord, Moyen-Orient: la double recomposition, Paris, La Documentation française, 2013-2014, p. 99.

2. Voir Ayyam Sureau, «La révolution égyptienne», Commentaire, 2011, $\mathrm{n}^{\circ}{ }_{134}$; JeanNoël Ferrié, «Dispositifs autoritaires et changements politiques. Les cas de l'Égypte et du Maroc", Revue internationale de politique comparée, 2012, vol. 19, $\mathrm{n}^{\circ} 4$; Pierre Vermeren, Maghreb, les origines de la révolution démocratique, Paris, Pluriel, 2011, 420 p.; Tahar Ben Jelloun, L'étincelle: révoltes dans les pays arabes, Paris, Gallimard, 2011; Masri Feki, Les révoltes arabes: géopolitique et enjeux, LevalloisPerret, Studyrama, 2011; Michäl Béchir Ayari et Vincent Geisser, Renaissances arabes, Ivry-sur-Seine, L'Atelier, 2011 ; Olivier Piot, La révolution tunisienne, Paris, Les petits matins, 2011; Antoine Basbous, Le tsunami arabe, Paris, Fayard, 2011; Sarah Ben Néfissa et Blandine Destremau (dir.), Protestations sociales, révolution civiles, Transformations du politique dans la méditerranée arabe, Revue du Tiers Monde, hors-série $\mathrm{n}^{\mathrm{o}}$ 2, 2011; Moncef Marzouki, L'invention d'une démocratie. Les leçons de l'expérience tunisienne, Paris, La découverte, 2013; Bilan géostratégique: le monde arabe en transition, Moyen-Orient, 2011 ; Bichara Khader, Le printemps arabe: un premier bilan, Paris, Syllepse, 2012; Revue française de science politique, vol. 62, $\mathrm{n}^{\mathrm{o}}$ 5-6, 2012; Printemps arabe et démocratie, Questions internationales, $\mathrm{n}^{\mathrm{o}} 53,2012$; Printemps arabes: comprendre les révolutions en marche, Mouvement des idées et des luttes, $\mathrm{n}^{\mathrm{0}} 66,2011$. 
pays $^{3}$. La dernière étude ayant une approche globale du constitutionnalisme arabe date de $2009^{4}$. Pourtant, si les approches thématiques ou étatiques restent essentielles, il est important de tenter de mettre en avant les tendances constitutionnelles globales qui semblent traverser l'ensemble de ces « révolutions». En effet, on assiste dans plusieurs pays à une transition démocratique plus ou moins rapide et poussée. On parle ainsi de "printemps arabe", de "révolution arabe» ou encore de «soulèvements populaires». Les causes de tels soulèvements sont multiples : pays autoritaires, libertés publiques réduites, pratiques politiques antidémocratiques, sociétés fortement inégalitaires socialement, souveraineté populaire confisquée, développement des réseaux sociaux, émergence d'une société civile. Ces logiques de ruptures ont débuté en 2010 en Tunisie, plongeant le pays dans une profonde crise politique. Ces événements vont avoir plusieurs conséquences, notamment sur le plan du droit public et plus précisément du droit constitutionnel. L'approche de cet article est, avant tout, juridique. Dès lors, nous ne nous attarderons pas sur les conséquences politiques de ces nouvelles mutations. Nous

3. Voir Ayman Mohamed Afify, «La résistance des juges en Égypte à la limitation du contrôle de constitutionnalité des lois électorales», RDP 2014, ${ }^{\circ}{ }^{1}$, p. 85 ; Nadia Bernoussi et Abderrahim Maglouhi, «Les chantiers de la bonne justice. Contraintes et renouveau », RFDC 2012, n 31, p. 479; Ferchichi Wahid «L'environnement dans la nouvelle constitution tunisienne du 27 janvier 2014 ", Rev. jur. env. ${ }^{\text {er }}$ janvier 2014, p. 215-218. Voir sur l'Égypte, François Sureau, «Égypte: une constitution en deux mondes ", Pouvoirs, 2014, vol. 2, n ${ }^{\circ} 149$; Stéphane Lacroix, «L’Égypte, entre révolution et contre-révolution ", dans Afrique du Nord, Moyen-Orient: l'échec du rêve démocratique, Paris, La Documentation française, 2014-2015, p. 35 ; Nathalie BernardMaugiron, «La constitution égyptienne de 2014: quelle réforme constitutionnelle pour l'Égypte?», RFDC 2015, $\mathrm{n}^{\circ} 103$; Ayman Mohamed Afify, "La constitution égyptienne de 2014: entre traditions et tendances révolutionnaires", RFDC 2015, $\mathrm{n}^{\mathrm{o}} 101$; Jean-Yves de Cara et Charles Saint-Prot, L'évolution constitutionnelle de l'Égypte, Paris, Karthala, 2014. Voir sur le Maroc, Omar Bendourou, «La nouvelle constitution marocaine du 29 juillet 2011 : le changement entre mythe et réalité », RDP 2012, p. 639; "Le Maroc», Pouvoirs, 2013, vol. 2, nº 145; Ahmed Bouachik et al. (dir.), La constitution marocaine de 2011, lectures croisées, Rabat, Remald 2012 ; Centre d'études internationales, La constitution marocaine de 2011: analyses et commentaires, Paris, LGDJ, 2012; Omar Bendourou, «La nouvelle constitution marocaine du 29 juillet 2011 », RFDC 2012, $n^{\circ} 91$; Sanae Kasmi, «Le statut de l'opposition au Maroc», RFD const. 2015, $\mathrm{n}^{0}$ 102, p. 429-452. Voir sur la Tunisie, Rafâa Ben Achour et Sana Ben Achour, "La transition démocratique en Tunisie: entre légalité constitutionnelle et légitimité révolutionnaire», RFDC 2012, ${ }^{\circ}$ 92, p. 715; Rafâa Ben Achour, "La constitution tunisienne du 27 janvier 2014", RFDC 2014, $\mathrm{n}^{\mathrm{o}} 100$, p. 783 ; Ferchichi Wahid, «L'environnement dans la nouvelle constitution tunisienne du 27 janvier 2014", Rev. jur. env. 2014, p. 215-218.

4. Thierry Le Roy, «Le constitutionnalisme : quelle réalité dans les pays du Maghreb», RFDC $2009, n^{\circ} 79$. 
nous interrogerons ici sur les caractéristiques des mutations et des transformations constitutionnelles en cours, sur leurs portées et leurs limites. Il ne s'agira pas d'être exhaustif, mais de tenter de dégager les grandes caractéristiques de ces transformations constitutionnelles. Nous assistons, en effet, à de vastes mutations constitutionnelles tendant à aller dans le sens d'une certaine démocratisation de ces régimes. Les spécificités de ces mouvements, ou tendances constitutionnelles, se manifestent, à notre avis, à travers une consolidation des droits fondamentaux (1) et un rééquilibrage entre les statuts des différents pouvoirs (2). Néanmoins ces nouveaux mouvements constitutionnels restent limités et ne peuvent faire l'économie de certaines critiques et réserves, imposant sûrement de nouvelles réformes (3).

\section{Vers une consolidation des droits fondamentaux}

La consolidation des droits fondamentaux apparaît véritablement comme une des tendances fortes de ces nouveaux mouvements constitutionnels. Elle se manifeste, semble-t-il, à travers trois variables : le renforcement des droits existants (1.1), la reconnaissance de droits nouveaux (1.2) et, enfin, la mise en place de nouveaux mécanismes de protection (1.3).

\subsection{La consolidation des droits existants}

La consolidation des droits existants constitue le premier mouvement observable et nous pouvons alors citer quelques exemples qui en témoignent. Il en est ainsi du droit à la sûreté. En Algérie, par exemple, dans un souci de protection, la réforme de 2016 a intégré le principe selon lequel « la détention provisoire doit être une mesure exceptionnelle dont les motifs, la durée et les conditions de prorogation sont définis par la loi» tout en précisant que le législateur doit punir les actes d'arrestation arbitraire. Au sein de la constitution égyptienne de 2012, dont les dispositions relatives à la sûreté seront reprises par celle de 2014, il est fait mention de «l'inviolabilité de la liberté personnelle». De même, la protection de la vie privée est confortée dans le sens où cette notion est explicitement énoncée et au fait que cette protection doit non seulement concerner le domicile et les correspondances, mais également les communications et les données personnelles. Cette approche extensive de la vie privée va dans le bon sens puisque conforme au droit international, tel le pacte international de 1966 relatif aux droits civils et politiques. La liberté de culte bénéficie également d'une nouvelle portée puisque, dorénavant, il est indiqué explicitement que celle-ci comprend aussi 
la construction de lieux de culte et qu'une telle liberté est absolue. La liberté de la presse, très sommaire à l'origine au sein des différents textes fondamentaux, va faire l'objet de développements substantiels. Ainsi, en Algérie, antérieurement à la réforme de 2016, la Constitution restait très timide sur la reconnaissance de certaines libertés. L'article 41 disposait, dans une formule lapidaire, que la liberté d'expression était "garantie au citoyen». La révision de 2016 fait preuve de plus de précision en faisant référence à la liberté « de la presse écrite, audiovisuelle » et à celle relative aux « réseaux d'information ». D'autre part, est posé le principe selon lequel le délit de presse «ne peut être sanctionné par une peine privative de liberté ». Cette démarche s'intègre dans la volonté d'adhérer aux principes posés par le droit international.

\subsection{La reconnaissance de nouveaux droits}

Non seulement nous constatons la consolidation des droits existant, mais nous remarquons aussi la reconnaissance de droits nouveaux. Il en est ainsi de la question de l'égalité homme/femme qui est une question centrale lorsqu'on connaît la situation de la femme dans un très grand nombre de ces pays ${ }^{5}$. Face à cette situation certains d'entre eux se sont engagés, il y a peu, vers l'égalité homme/femme. Il s'agit là d'une

5. Dans son rapport de 2015 la Banque mondiale a pu mettre en évidence les discriminations qui persistent dans 19 économies de la région Moyen-Orient et Afrique du Nord, et pour la première fois, le Bahreïn, Djibouti, l'Iraq, Malte et le Qatar. La région Moyen-Orient et Afrique du Nord est celle qui possède le plus de lois limitant l'activité économique des femmes. Les dispositions juridiques qui interdisent aux femmes mariées de devenir chef de famille, de faire une demande de passeport ou d'être embauchées sans l'autorisation de leur mari limitent participation à la vie économique. Parmi les économies de la région, l'Algérie, Djibouti et Malte sont les seuls qui autorisent la femme - au même titre que l'homme - à transmettre sa nationalité à ses enfants et à son conjoint. Par ailleurs, 18 pays imposent des restrictions sur le type d'emplois que les femmes peuvent occuper, cette situation entraînant de forts écarts de salaire avec les hommes. La région abrite 11 des 15 économies les plus restrictives au monde en matière de réglementations concernant les femmes salariées et entrepreneurs, à savoir l'Arabie Saoudite, la Jordanie, l'Iran, le Yémen, l'Iraq, le Bahreïn, les Émirats arabes unis, Oman, la Syrie, le Qatar et le Koweï. L'Afghanistan, le Soudan, la Mauritanie et le sultanat de Brunei font également partie de la liste. Le statut d'infériorité des femmes sur le plan juridique entraîne un taux de scolarisation des filles plus faible dans le secondaire, un nombre moins important de femmes chefs d'entreprise ou salariées et un écart salarial plus grand entre les hommes et les femmes. Dans les pays où aucune loi ne les protège contre les violences conjugales, les femmes voient leur espérance de vie raccourcie (Rapport Les femmes, l'entreprise et le droit, rapport de la Banque mondiale, septembre 2015). 
véritable avancée s'intégrant dans un processus qu'on peut qualifier de rapide lorsqu'on le compare aux lentes évolutions ayant eu lieu dans certains pays occidentaux ${ }^{6}$. Nous pouvons citer, à ce titre, le Maroc où, suite à la révision constitutionnelle du $1^{\text {er }}$ juillet 2011, l'article 19 de la Constitution dispose, dorénavant, que l'homme et la femme jouissent, à égalité, des droits et libertés à caractère civil, politique, économique, social culturel ou environnemental. Ce principe d'égalité concerne aussi les fonctions électives où le législateur est invité à intervenir. Cette volonté de promouvoir les droits des femmes s'intègre dans le droit fil du droit international et plus précisément de la convention des Nations unies en date du 18 décembre 1979 sur l'élimination de toutes les formes de discriminations à l'égard des femmes. En Tunisie, la question de l'égalité entre homme et femme va trouver, pour la première fois, une assise constitutionnelle au sein de la Constitution de 2014. En effet, l'article 11 dispose que l'État s'engage à réaliser l'égalité entre les femmes et les hommes dans les domaines des droits civils, politiques, économiques, sociaux et culturels, conformément aux dispositions du nouveau texte constitutionnel. L'État doit également s'engager à prendre les mesures nécessaires afin d'assurer une juste représentation des femmes au sein du parlement. Il est intéressant d'observer qu'il ne s'agit pas seulement de reconnaitre de nouveaux droits à la femme, mais la loi fondamentale fait obligation à l'État d'agir en ce sens. La nouvelle loi fondamentale garantit aussi le droit pour toutes femmes d'accéder, sans discrimination, aux emplois publics et aux hautes fonctions de direction au sein de l'administration publique d'État ainsi que dans les institutions judiciaires. Afin de donner toute réalité à ce principe, les députés tunisiens ont voté le 15 juin 2016 l'article 49 du projet de loi sur les élections municipales. En vertu de cette loi, chaque liste devra comporter un nombre égal d'hommes et de femmes selon le système d'alternance, et au moins la moitié des listes électorales présentées par les partis politiques devra être présidé par des femmes. La constitution syrienne du 26 février 2012 va également dans le sens d'un renforcement des droits des femmes. Il est reconnu dans ce sens l'égalité des citoyens devant la loi et cela sans discrimination de sexe. Enfin, la réforme constitutionnelle en Mauritanie, en date de 2012, reconnaît, pour la

6. Ce processus est rapide pour des pays qui ont obtenu leur indépendance il y a peu. En comparaison, en France ce n'est qu'en 1938 qu'une loi est venue supprimer l'incapacité juridique de la femme; le droit de vote n'est reconnu qu'en 1944 et ce n'est qu'en 1965 que les femmes mariées peuvent exercer une profession sans l'autorisation de leur mari. 
première fois, l'égalité entre homme et femme en ce qui concerne les mandats électoraux et les fonctions électives.

Un autre domaine semble parcouru par cette consolidation. Il s'agit de celui de la procédure pénale. Ainsi, suite à la révision constitutionnelle marocaine de 2011, plusieurs dispositions concernent la sûreté de l'individu. La détention arbitraire ou secrète et la disparition forcée sont considérées désormais comme des crimes. De même, toute personne détenue doit dorénavant être informée immédiatement d'une façon qui lui soit compréhensible des motifs de sa détention et de ses droits, dont celui de garder le silence qui est un des éléments de la présomption d'innocence. Elle doit bénéficier aussi, au plus tôt, d'une assistance juridique. L'article 23 reconnaît également explicitement le principe de la présomption d'innocence tel que reconnu par le droit international ${ }^{7}$. La constitution syrienne du 26 février 2012 témoigne de cette évolution favorable dans le domaine touchant les droits de la défense. Par exemple, la loi fondamentale reconnaît le droit pour toute personne de bénéficier d'un procès régulier comme le droit de faire appel et de plaider devant un jury. D’autres dispositions sont relatives à la procédure pénale. Il est reconnu dans ce sens que, à l'avenir, lors de son arrestation la personne arrêtée doit être informée de ses droits. Il est fait interdiction de garder une personne sous le contrôle de l'autorité administrative, si ce n'est en vertu d'un ordre de l'autorité judiciaire compétente. Il s'agit ici de condamner les détentions arbitraires contraires à l'article $9 \mathrm{du}$ Pacte international relatif aux droits civils et politiques du 16 décembre 1966. L'autorité judiciaire apparaît ici, comme c'est le cas en France, comme la gardienne des libertés. Enfin, les personnes se voient octroyer un droit à l'indemnisation si le verdict est erroné et la peine appliquée. La constitution tunisienne en date de 2014 témoigne aussi de cette volonté de rationaliser davantage la procédure afin de mieux protéger les libertés individuelles. En effet, face aux abus de l'ancien régime le nouveau texte apporte des garanties telles que l'interdiction de faire l'objet d'une arrestation (sauf en cas de flagrant délit ou sur la base d'une décision judiciaire), le droit de se faire représenter par un avocat. La durée de la garde à vue et de la détention doit être, en outre, explicitement définie

7. Voir notamment l'art. 16 de la Charte arabe des droits de l'homme; l'art. 7 de la Charte africaine des droits de l'homme et des peuples; l'art. 11 de la Déclaration universelle des droits de l'homme; l'art. 14-2 du Pacte des Nations unies relatif aux droits civils et politiques; l'art. 48 de la Charte des droits fondamentaux de l'UE; l'art. 6 de la Convention européenne de sauvegarde des droits de l'homme et des libertés fondamentales. 
par la loi afin de limiter tout arbitraire. C'est ainsi que le 16 février 2016 le parlement tunisien a adopté un projet de la loi qui réduit la durée de la garde à vue, permet l'accès à un avocat dès le début de celle-ci et réaffirme le droit des détenus à voir un médecin. La loi fondamentale ajoute que tout détenu a droit à un traitement humain qui préserve sa dignité. Enfin, le nouveau texte fondamental indique que la loi doit garantir le double degré de juridiction (art. 108). La constitution égyptienne de 2012, puis celle de 2014, illustrent aussi cette consolidation de la liberté personnelle comme en témoignent plusieurs dispositions. C'est ainsi que l'interrogatoire d'une personne ne peut débuter qu'en présence de son avocat. S'il n'a pas d'avocat, un avocat devra alors être commis d'office. De plus, toute personne arrêtée ou détenue aura le droit de s'en plaindre devant le tribunal qui devra alors trancher dans un délai d'une semaine limitant ainsi les détentions arbitraires dans leur durée. Dans le cas contraire, la personne doit être remise immédiatement en liberté. Enfin, la Constitution de 2014 reprend le principe de l'inviolabilité du domicile tout en encadrant les atteintes éventuelles en précisant que les perquisitions ne peuvent se faire qu'à la suite d'un mandat judiciaire motivé devant préciser, dorénavant, le lieu, le moment et le but de celles-ci.

La liberté n'est pas la seule valeur faisant l'objet d'une attention particulière. Il en est de même pour les droits collectifs qui conditionnent la vitalité de la société civile et, au-delà, la démocratie elle-même. C'est ainsi que la révision constitutionnelle du 26 février 2012 relative à la Syrie reconnaît et garantit, pour la première fois, la liberté de former des associations et des syndicats. La nouvelle constitution tunisienne, dans son article 37, consacre la liberté de rassemblement et de manifestation. Seront également reconnus le droit de grève et la liberté syndicale. Il s'agissait là de l'une des revendications des syndicats salariés tunisiens qui ont joué un rôle non négligeable, voir déterminant, lors de la « révolution ». La reconnaissance de tels droits collectifs s'intègre dans la volonté de se conformer au droit international. Mais il faut bien garder à l'esprit que si l'on peut penser, dans un premier temps, qu'il s'agit de droits octroyés, il s'agit avant tout de droits qui ne sont que le produit de luttes sociales, de revendications comme celles qui se sont déroulées lors du printemps arabe en Tunisie au cours des années 2010-2011.

Enfin, on observe que les droits sociaux ont fortement imprégné ces nouveaux mouvements constitutionnels. À l'État régulateur semble vouloir se substituer l'État protecteur. En effet les dirigeants arabes prennent conscience que la stabilité d'un pays ne peut se faire en laissant de côté une grande partie de la population, la plus fragile socialement. Les 
différents soulèvements auxquels on a pu assister tant en Tunisie, qu'en Égypte ou encore au Maroc ont trouvé leur fondement, notamment, sur le terrain de la question sociale $(15,5 \%$ des Tunisiens se situent en deçà du taux de pauvreté, $26 \%$ des Égyptiens ${ }^{8}$ et $20 \%$ des Marocains). La question sociale a véritablement intégré les constitutions arabes postrévolutionnaires. Il en est ainsi en Égypte avec la reconnaissance du droit au logement qui apparaît comme une nouveauté. En effet, la Constitution de 2014, dans le sillage de celle de 2012, précise que l'État garantit aux citoyens le droit à un logement "décent, sûr et sain, de manière à préserver la dignité humaine et à réaliser la justice sociale». Ces dispositions sociales s'intègrent dans le sillage des dispositions de la Déclaration universelle des droits de l'homme de 1948 ou de celles du Pacte international relatif aux droits économiques, sociaux et culturels de 1966. La protection des droits de l'enfant fait également l'objet d'une prise de conscience. La révision constitutionnelle algérienne, par exemple, en février 2016, dispose que la famille, la société et l'État doivent protéger les droits de l'enfant. L'État doit prendre en charge les enfants abandonnés ou sans affiliation et réprimer la violence à leur encontre. De nouvelles dispositions précisent même que, sous peine de poursuites, les parents ont l'obligation d'assurer l'éducation de leurs enfants. Ces dispositions vont dans le bon sens en se rapprochant des principes proclamés tant par la Convention internationale des droits de l'enfant en date de 1989 que par la Charte africaine des droits et du bien-être de l'enfant signée en 1990 dans le cadre de l'Organisation de l'unité africaine ratifiée par l'Algérie. On peut également remarquer que l'accès aux soins est une question qui a, semble-t-il, pénétrer les différents textes fondamentaux. La plupart des pays arabes connaissent une situation sanitaire difficile ${ }^{9}$. En outre, derrière la question de l'accès aux soins pointe, notamment, la question de l'espérance de vie et donc celle de l'égalité devant la mort. C'est ainsi que, se situant dans le mouvement de la Constitution de 2012, la constitution égyptienne de 2014 souligne que l'État s'engage d'une part, à allouer un pourcentage des dépenses publiques à la santé qui ne peut pas être inférieur à $3 \%$ du PIB et, d'autre part, à mettre en place un système complet de protection de la santé pour tous les Égyptiens, couvrant toutes les maladies ${ }^{10}$. La nouvelle

8. http://www.slateafrique.com/617161/egypte-au-caire-des-milliers-dappartementsvides-et-des-pauvres-mal-loges.

9. La mortalité infantile est de 24/1 ooo au Maroc et le montant annuel consacré aux soins pour chaque habitant est seulement de 189 \$. 
constitution tunisienne ira dans le même sens en reconnaissant à chacun «le droit à la santé ».

\subsection{La mise en place de nouveaux mécanismes de protection}

Les droits fondamentaux ne sauraient trouver une pleine effectivité sans mécanismes de contrôle et de mise en œuvre. Les nouveaux mouvements constitutionnels arabes semblent aller dans le bon sens et témoignent d'une volonté de donner toute leur portée à ces droits, c'est-à-dire leur octroyer une certaine réalité politique. Il y a une réelle volonté de sortir du déclaratif et de ses limites. On peut constater que le nouveau constitutionnalisme arabe se tourne dans différentes directions afin d'assurer cette effectivité. C'est ainsi que la nouvelle constitution tunisienne, comme la dernière réforme constitutionnelle algérienne, pose le principe selon lequel «le préambule fait partie intégrante » de la constitution. Il s'agit là d'éviter toute interprétation restrictive de la constitution de la part du juge constitutionnel, mais plus encore d'inciter ce juge à faire preuve d'audace. La protection des droits fondamentaux, et donc leur effectivité, passe aussi, dans l'avenir, par l'obligation constitutionnelle faite aux États arabes d'accomplir des actes positifs. Les constituants sont venus donner de véritables directives au législateur. Nous pouvons citer la révision constitutionnelle marocaine où il est précisé que «l'État marocain œuvre à la réalisation de la parité entre les hommes et les femmes». "Il est créé, à cet effet, une Autorité pour la parité et la lutte contre toutes formes de discrimination.» La nouvelle constitution égyptienne mentionne que l'État doit s'engager à mettre en œuvre un programme de logement qui vise à atteindre l'équilibre entre le taux de croissance de la population et les ressources disponibles, en maximisant l'investissement dans l'énergie humaine et en améliorant ses caractéristiques, dans le cadre de la réalisation du développement durable. Il est précisé explicitement que l'État doit garantir aux citoyens le «droit à un logement décent, sûr et sain, de manière à préserver la dignité humaine et à réaliser la justice sociale». Il doit élaborer pour cela un plan national pour le logement qui respecte la spécificité environnementale et garantisse dans sa mise en œuvre les initiatives individuelles et coopératives. Il doit également mettre en place un plan national global pour résoudre le problème des bidonvilles, qui comprenne la fourniture d'infrastructures

10. Il est précisé que ce pourcentage devra augmenter progressivement pour atteindre les ratios mondiaux. 
et d'équipements, et l'amélioration de la qualité de la vie et de la santé publique. Il doit aussi garantir les ressources nécessaires à sa mise en œuvre dans un laps de temps précis. Le nouveau texte tunisien ne se limite pas à reconnaître le droit à la santé, mais donne les grandes lignes à suivre afin d'en assurer l'effectivité puisque la nouvelle loi fondamentale souligne que "pour mener à bien cette mission [assurer le droit à la santé] il est fait obligation à l'État d'une part, d'assurer la prévention et les soins sanitaires à tout citoyen en fournissant les moyens nécessaires pour garantir la sécurité et la qualité des services de santé et, d'autre part, mettre en place une couverture sociale». «Il doit assurer à ce titre la gratuité des soins pour les personnes sans soutien et à faible revenu.» Cette volonté de donner une certaine intensité à la protection des droits et libertés individuels s'exprime aussi par la mise en place de mécanismes de contrôle constitutionnels par voie d'exception comme en Syrie (2012), en Tunisie (2014), au Maroc (2011) ou encore en Algérie (2016). Certains auteurs, comme Léon Duguit, militait fortement pour la mise en place d'un tel système en France. Celui-ci écrivait ainsi dans les années 1920: «Les ÉtatsUnis ont l'honneur d'avoir constitué un système qui assure [...] la réalisation de cet idéal [la protection des libertés individuelles à travers le mécanisme de l'exception d'inconstitutionnalité ${ }^{11}$ ].» Il ajoutait : «Je n'hésite pas à dire que c'est un devoir pour tout tribunal de recevoir l'exception d'inconstitutionnalité et de refuser l'application dans l'espèce qui lui est soumise d'une loi qui viole directement une disposition inscrite dans la constitution ${ }^{12}$." Le contrôle constitutionnel par voie d'exception constitue un second filtre garant des droits fondamentaux des citoyens. Ce contrôle est d'autant plus important que le pouvoir législatif arabe ne possède pas forcément de maturité en matière de culture démocratique, même si celle-ci tient une place grandissante. On remarque aussi que, de manière générale, ces nouveaux mouvements constitutionnels reconnaissent une fonction protectrice au juge face à tout éventuel arbitraire de l'administration. Ainsi, la constitution marocaine, suite à sa dernière révision, souligne que les partis politiques ne peuvent être suspendus ou dissous par les pouvoirs publics que sur la base « d'une décision de justice » (art. 9). Enfin, on assiste dans un certain nombre de constitutions à la mise en place d'organes indépendants

11. Léon Duguit, Souveraineté et liberté, Paris, La mémoire du droit, 2002, p. 200.

12. Léon Duguit, Leçons de droit public général faites à la Faculté de droit de l'Université égyptienne pendant les mois de janvier, février et mars 1926, Paris, De Boccard, 1926, p. 292. 
ayant pour mission d'assurer la promotion ainsi que la protection des droits de l'homme. En Mauritanie, en 2012, a été créée la Commission nationale des droits de l'homme, en 2014, en Tunisie une Commission de la communication audiovisuelle, ainsi qu'une Commission des droits de l'homme ont vu le jour, de même qu'un Conseil national des médias en Égypte ayant pour mission de garantir la liberté de la presse. Au Maroc, a été créée une autorité pour la parité et la lutte contre toutes formes de discriminations entre femmes et hommes. Enfin, en 2016, en Algérie, la révision constitutionnelle a donné naissance à un Conseil national des droits de l'homme.

On remarque que les constituants arabes sont aussi venus assurer la protection des droits fondamentaux en les excluant de toute procédure de révision constitutionnelle tendant éventuellement à les remettre en cause. La constitution tunisienne dispose ainsi, aujourd'hui, dans son article 49, qu' "aucun amendement ne peut porter atteinte aux droits de l'Homme et aux libertés garantis par la présente Constitution ». La réforme ayant concerné le Maroc exclut également de toute modification «les acquis en matière de libertés et de droits fondamentaux» inscrits dans la constitution.

Enfin, certains constituants arabes n'hésitent pas à sanctionner pénalement l'atteinte à certains droits fondamentaux. C'est ainsi que la constitution égyptienne souligne aujourd'hui que le fait de refuser un traitement médical à toute personne, en cas d'urgence ou de risque mortel, est un crime.

\section{La recherche d'un rééquilibrage des pouvoirs}

La volonté de tendre vers un meilleur équilibre des pouvoirs semble constituer une autre caractéristique de ces mutations constitutionnelles. Cette nouvelle option trouve son fondement dans l'état du droit constitutionnel antérieur, tout comme la pratique du pouvoir qui plaçait le pouvoir exécutif dans la toute-puissance. Afin d'assurer ce rééquilibrage, il semble que les constituants soient allés dans 3 grandes directions: la valorisation du parlement (2.1); la rationalisation du pouvoir exécutif (2.2); la rénovation du statut du pouvoir judiciaire (2.3).

\subsection{La valorisation du parlement}

Cette volonté de revaloriser le pouvoir législatif trouve son fondement dans la prédominance de l'exécutif tant en droit que dans une certaine pratique du pouvoir. Cette nouvelle donne constitutionnelle semble aussi trouver un fondement dans une tendance plus globale du droit 
constitutionnel. On pense ainsi aux réformes constitutionnelles ayant concerné plusieurs pays dont la Lettonie le 4 décembre 1997, l'Ukraine le 8 décembre 2004, le Cambodge le 9 mars 2006, la Norvège le 30 mars 2007, la France le 23 juillet 2008. Il s'agit là d'un retour à un principe fondamental: le parlement comme source de la souveraineté. Néanmoins, il semble que cette revalorisation ne doive pas être poussée à l'extrême entraînant un régime d'assemblée qui a pu être dénoncé, notamment, par Gaston Jèze sous de la III ${ }^{\mathrm{e}}$ République $^{13}$.

La revalorisation du parlement se matérialise de façons très diverses.

Il y a, dans un premier temps, une consolidation du contrôle politique sur le pouvoir exécutif. Ainsi, afin que le parlement ne soit plus qu'une simple chambre d'enregistrement, la nouvelle constitution égyptienne souligne que, dorénavant, les lois sont votées à la majorité absolue des membres présents, qui doit être égale au tiers au moins des membres de la chambre. La réforme constitutionnelle de 2012 en Mauritanie (mais également la réforme constitutionnelle au Bahreïn en date de 2012) impose au Premier ministre, au plus tard un mois après la nomination du gouvernement, de présenter son programme devant l'Assemblée nationale et d'engager la responsabilité de son gouvernement sur ce programme $^{14}$ (art. 42). De même, le sultan Qabus d'Oman a accordé, en octobre 2011, des réformes en amendant la loi fondamentale du sultanat pour renforcer les pouvoirs du parlement qui doit désormais se voir communiquer par le Conseil des ministres les projets de loi pour examen et révision, et cela, avant de les transmettre au sultan. Ce contrôle politique passe aussi par un parlement mieux informé. C'est dans ce sens que la réforme marocaine, en date de 2011, impose au gouvernement de soumettre annuellement au parlement une loi de règlement de la loi

13. Gaston Jèze, Les principes généraux du droit administratif, t. 1, La technique juridique du droit public français, Paris, Dalloz, 2005, p. 210.

14. Jusque-là, cette procédure était prévue par la constitution, mais à titre purement facultatif. En imposant la question de confiance «préalable» au Premier ministre, cette réforme réduit la marge de manœuvre du président de la République qui doit désormais choisir un Premier ministre (et un gouvernement) bénéficiant de l'appui de la majorité au parlement. Mais le président de la République conserve l'ensemble de ses autres prérogatives. À travers ce mécanisme, il y a incontestablement une «dose» de parlementarisme qui est introduite dans le système politique mauritanien. Cela pourrait induire une modification des rapports institutionnels entre le président de la République et le Premier ministre d'une part, et entre le pouvoir exécutif et le pouvoir législatif, d'autre part. et, au-delà, une «lecture parlementaire» des autres dispositions constitutionnelles. À cet égard, la pratique constitutionnelle subséquente sera déterminante. 
de finances portant sur l'exercice précédent ${ }^{15}$. Cette loi doit notamment inclure le bilan des budgets d'investissement dont la durée est arrivée à échéance. De plus, le chef du gouvernement doit présenter devant le parlement un bilan d'étape de l'action gouvernementale, à son initiative ou à la demande du tiers des membres de la chambre des représentants ou de la majorité des membres de la chambre des conseillers. Une séance annuelle est, en outre, réservée au parlement pour discuter et évaluer les politiques publiques. On remarque également que les commissions concernées dans chacune des deux chambres peuvent demander à auditionner les responsables des administrations et des établissements et entreprises publics, en présence et sous la responsabilité des ministres dont ils relèvent. Ce contrôle politique se matérialise par la consolidation du statut des commissions d'enquêtes parlementaires puisqu'au Maroc, par exemple, on assiste à un élargissement de leur champ d'investigation qui concerne, depuis 2011, entre autres «la gestion des services, entreprises et établissements publics ${ }^{16} »$. Enfin, un dernier élément semble témoigner du renforcement d'un tel contrôle politique. Il se matérialise à travers la responsabilité accrue du pouvoir exécutif. C'est ainsi que, par exemple, la nouvelle constitution tunisienne permet au pouvoir législatif de retirer sa confiance non seulement au gouvernement, mais également à un ministre plus particulièrement (art. 97). Le nouveau texte pose aussi le principe selon lequel «le Gouvernement est responsable devant l'Assemblée des représentants du peuple » (antérieurement il était responsable devant le président de la République, art. 95). Le contrôle politique va même jusqu'à donner la possibilité au pouvoir législatif de remettre en cause la responsabilité politique du chef de l'État, comme en témoigne la réforme constitutionnelle tunisienne (art. 88).

En observant les nouvelles tendances constitutionnelles arabes, on remarque que la revalorisation du pouvoir législatif passe aussi par la reconnaissance d'une plus grande autonomie. L'exemple de la réforme tunisienne est révélateur de cette volonté. Ainsi, depuis 2014, le nouveau texte fondamental précise que l'Assemblée des représentants du peuple

15. La réforme constitutionnelle de février 2016 en Algérie va dans le même sens. En effet, l'art. 84 disposait auparavant que " le gouvernement présent[ait] annuellement à l'Assemblée Populaire Nationale une déclaration de politique générale ». Afin que cette présentation puisse revêtir un caractère obligatoire le constituant utilise en 2016 la formule "doit présenter».

16. Dans le même sens, la nouvelle constitution égyptienne de 2014 souligne que les commissions d'enquêtes parlementaires peuvent également concerner « une entreprise publique» et non plus seulement un service administratif ou un organisme public. 
jouit de «l'autonomie administrative et financière » et que l'État doit mettre à la disposition de l'assemblée les ressources humaines et matérielles nécessaires à chaque député pour le bon exercice de ses fonctions. En effet, un parlement sans moyen financier ne pourra mener à bien sa fonction, comme au Tchad par exemple, où l'on peut parler de parlement «fantôme».

L'élargissement de la compétence matérielle du pouvoir législatif permet aussi d'illustrer cette revalorisation. Ainsi au Maroc, suite à la dernière réforme constitutionnelle, le domaine de la loi est élargi, intégrant à l'avenir le statut de la famille ou encore l'état civil ${ }^{17}$. Il en a été de même en Tunisie où ont été intégrés dans le domaine de compétence de la loi les traités commerciaux, ceux relatifs à l'organisation internationale ou aux frontières de l'État, ceux portant engagement financier de l'État ou concernant le statut des personnes, ou encore portant modification de dispositions à caractère législatif.

Enfin, on constate que le statut des sessions parlementaires est consolidé, marquant ainsi un pouvoir accru du parlement et sa valorisation. Dans ce sens, en Algérie, depuis 2016, la session ne peut être inférieure à 10 mois contre 8 mois antérieurement. En Mauritanie, la réforme de 2012 pose le principe de deux sessions parlementaires de 4 mois chacune contre 2 mois auparavant. Ces réformes témoignent d'une volonté d'avoir un parlement plus présent et donc plus actif.

\subsection{La rationalisation du pouvoir exécutif}

La référence à la notion de rationalisation est généralement employée lorsqu'on traite des réformes ayant pour objet de réduire les prérogatives du pouvoir législatif vis-à-vis de l'exécutif dans le cadre d'un régime politique et cela afin de tendre vers un certain équilibre des pouvoirs.

17. Mais on trouve également le droit des sociétés et des coopératives, les droits réels et les régimes des propriétés immobilières publique, privée et collective, le régime des transports, les relations de travail, la sécurité sociale, les accidents de travail et les maladies professionnelles, le régime des banques, des sociétés d'assurances et des mutuelles, le régime des technologies de l'information et de la communication, l'urbanisme et l'aménagement du territoire, les règles relatives à la gestion de l'environnement, à la protection des ressources naturelles et au développement durable, le régime des eaux et forêts et de la pêche, la détermination des orientations et de l'organisation générale de l'enseignement, de la recherche scientifique et de la formation professionnelle, la création des établissements publics et de toute autre personne morale de droit public, la nationalisation d'entreprises et le régime des privatisations. En outre, le parlement est habilité à voter des lois cadres concernant les objectifs fondamentaux de l'activité économique, sociale, environnementale et culturelle de l'État. 
Ici, l'emploi de cette notion traduit la volonté de tendre vers un meilleur équilibre entre les deux têtes de l'exécutif. Cette volonté de rationalisation dans le mouvement constitutionnel arabe se manifeste d'une part à travers un certain renforcement des prérogatives du gouvernement (2.2.1) et, d'autre part, par une certaine réduction, minoration, voir encadrement des pouvoirs du chef de l'État (2.2.2).

\subsubsection{Le statut renforcé du gouvernement}

Les régimes arabes sont marqués, avant tout, par la puissance et la prépondérance du pouvoir exécutif. Il n'y a qu'à se référer à la présidence de Ben Ali, celle d'Hosni Moubarak ou encore à celle de l'actuel chef d'État Abdelaziz Bouteflika. Dans le cadre de ces régimes, le pouvoir exécutif, même s'il semble dualiste au niveau formel, n'en reste pas moins concentré entre les mains du chef de l'État. Les "printemps arabes » sont, semble-t-il, venus impulser une nouvelle dynamique institutionnelle en renforçant le statut du gouvernement afin d'assurer une certaine émancipation de celui-ci vis à vis du chef de l'État. On remarque que la rénovation de ce statut s'exprime de multiples manières. On observe ainsi qu'il y a une volonté d'assurer une certaine autonomisation de la fonction gouvernementale. Dans ce sens, par exemple, la réforme constitutionnelle marocaine vient expressément poser le principe selon lequel le gouvernement "exerce le pouvoir exécutif » alors que la constitution antérieure restait silencieuse à ce sujet. La nouvelle constitution tunisienne, elle, mentionne que le pouvoir exécutif est désormais exercé non seulement par le président de la République, mais également par le gouvernement présidé par "un chef de Gouvernement», rompant ainsi avec le principe de la compétence exclusive du chef de l'État dans ce domaine. Cette volonté d'autonomisation se retrouve directement au sein même du nouvel article 91 où il est mentionné, depuis, que «le chef du Gouvernement détermine la politique générale de l'État, conformément aux dispositions de l'article 77 , et veille à sa mise en œuvre ». Ceci est une réelle avancée dans la reconnaissance du rôle du gouvernement lorsque l'on sait que la constitution antérieure mentionnait, à l'avantage du président de la République, que «le gouvernement veill[ait] à la mise en œuvre de la politique générale de l'État, conformément aux orientations et aux options définies par le président de la République». Cette autonomisation passe aussi par le fait, de la part du constituant, de préciser aujourd'hui les domaines de compétence du gouvernement, comme en témoigne la nouvelle constitution marocaine qui vient définir expressément les domaines dans lesquels statue le gouvernement tels 
que la politique générale de l'État, les politiques publiques, les politiques sectorielles, l'engagement de la responsabilité du gouvernement devant la chambre des représentants, les questions d'actualité liées aux droits de l'homme et à l'ordre public... Il faut noter que le renforcement du statut gouvernemental ne se matérialise pas seulement à travers une plus grande indépendance vis-à-vis du chef de l'État, mais également par l'attribution de nouvelles prérogatives. C'est ainsi que la réforme algérienne de février 2016 prévoit qu'en cas de persistance de désaccords entre les deux chambres, le gouvernement peut demander à l'Assemblée populaire nationale de statuer définitivement. En Tunisie, alors qu'antérieurement à la Constitution de 2014 l'exécution des lois revenait au chef de l'État, elle est depuis de la compétence du gouvernement. En Égypte, la Constitution de 2014 reconnaît au gouvernement le droit d'être à l'initiative des lois.

Il faut noter que ce n'est pas seulement le gouvernement dans son ensemble qui voit son statut consolidé. En effet, le chef du gouvernement, lui-même, voit son statut revalorisé afin de lui permettre d'exister face au chef de l'État. On observe ainsi, par exemple, que le nouveau texte tunisien met l'accent sur le fait que le chef du gouvernement "dirige» l'administration et "conclut» les traités internationaux à caractère technique. Depuis 2014, il lui revient de "présider» le Conseil des ministres, alors qu'auparavant celui-ci était présidé par le chef de l'État. Il a également compétence aujourd'hui à révoquer un ou plusieurs membres du gouvernement (art. 92). Afin de protéger le domaine de compétence entre le chef du gouvernement et le chef de l'État, le nouveau texte constitutionnel a même prévu que les conflits de compétences éventuels entre ces derniers sont soumis à la Cour constitutionnelle, à la demande de la partie la plus diligente et la cour doit trancher le conflit dans un délai limité, c'est-à-dire 1 semaine (art. 110). En Algérie, depuis la réforme de février 2016, il revient au chef du gouvernement de "présider les réunions du Gouvernement et de signer les décrets exécutifs ». La réforme constitutionnelle marocaine reconnaît l'autorité du Premier ministre sur les différents ministres et secrétaires d'État afin d'autonomiser les ministres de l'influence du palais. En effet, l'article 93 dispose en ce sens que « les ministres accomplissent les missions qui leur sont confiées par le Chef du Gouvernement ».

\subsubsection{La réduction des pouvoirs du chef de l'État}

Lorsqu'on regarde les grandes évolutions constitutionnelles touchant le monde arabe ces dernières années, on constate également une certaine 
volonté de réduire les prérogatives du chef de l'État et de limiter la concentration du pouvoir. Ce nouveau mouvement constitutionnel se traduit de multiples façons ${ }^{18}$. Nous pouvons remarquer, par exemple, qu'il y a une réelle volonté d'encadrer les pouvoirs présidentiels en temps de crise. C'est ainsi que la réforme constitutionnelle algérienne de 2016 conditionne la déclaration de l'état d'exception par le chef de l'État à la consultation, au préalable, du président du Conseil de la nation, du président de l'Assemblée populaire nationale et du président du Conseil constitutionnel. En outre, le Haut Conseil de sécurité et le Conseil des ministres doivent être entendus. Allant plus loin, on remarque que la constitution égyptienne de 2012, comme celle de 2014, met fin aux pouvoirs exceptionnels du chef de l'État en temps de crise (ancien art. 74 de la Constitution de 1971). Cette volonté de délimiter le pouvoir présidentiel se matérialise aussi par la mise en place d'un meilleur contrôle du droit de dissolution. Par exemple, au sein de la constitution égyptienne, si le chef de l'État possède le droit de dissolution, le décret de dissolution doit être «motivé » et pris «à la suite d'un référendum populaire». Au Bahreïn, avant toute dissolution de l'Assemblée nationale, le roi devra désormais consulter les présidents de la chambre, l'assemblée consultative et de la Cour constitutionnelle, alors qu'auparavant seul était sollicité l'avis (non contraignant) du Premier ministre. Cette limitation du pouvoir du chef de l'État apparait aussi au niveau des relations internationales qui, souvent, s'avèrent être un domaine "réservé», un domaine de souveraineté présidentielle. Il y a ici une intention, semble-t-il, de rompre avec ce principe. Ainsi, par exemple, en Égypte alors que la Constitution de 1971 permettait au chef de l'État de ratifier des traités sans l'intervention du pouvoir législatif, celle de 2014 met fin à cette possibilité, la chambre des représentants devant intervenir et, pour certains traités, ces derniers devant même être soumis au vote des citoyens. En outre, la possibilité pour le chef de l'État d'envoyer des troupes combattre à l'extérieur n'est plus soumise à un simple vote à la majorité de la chambre, mais à une majorité qualifiée des deux tiers de ses membres. Dans le même sens, la réforme algérienne a élargi les domaines des accords internationaux devant faire

18. Il faut également noter que la durée du mandat présidentiel va dans le sens d'une restriction. C'est ainsi que la réforme algérienne souligne que le président de la République est rééligible une seule fois et qu'aucune révision constitutionnelle ne peut porter atteinte à ce principe. Dans le même sens, la constitution révolutionnaire égyptienne de 2014 pose le principe d'un mandat de 4 ans renouvelable une fois contre 6 ans sans restriction de renouvellement dans la constitution de 1971. 
l'objet d'une "approbation express» par chacune des deux chambres $\mathrm{du}$ parlement. Ces domaines concernent depuis 2016, par exemple, les accords bilatéraux ou multilatéraux relatifs aux zones de libre-échange, aux associations et aux intégrations économiques. Enfin, les nouvelles tendances du constitutionnalisme arabe se caractérisent aussi par la volonté de réduire le pouvoir politique du chef de l'État dans le cadre des différentes nominations qu'il est amené à faire. En Égypte, par exemple, depuis 2014, si le président de la République peut procéder à un remaniement ministériel, c'est après consultation du Premier ministre et approbation de la chambre des représentants par un vote à la majorité absolue des membres présents, représentant au moins un tiers des membres de la chambre. En Algérie, la nomination du Premier ministre doit intervenir après consultation de la majorité parlementaire (art. 77). $\mathrm{Au}$ Maroc, le nouveau texte constitutionnel dispose que le roi doit nommer le chef du gouvernement au sein du parti politique arrivé en tête des élections des membres de la chambre des représentants (art. 47).

\subsection{Le pouvoir judiciaire : un statut rénové}

La capacité du pouvoir judiciaire à mener à bien sa mission est garante de toute démocratie. Dans ce sens Dominique Rousseau voit dans la justice «un pouvoir de la démocratie» et le professeur El Maslouhi considère que «l'indépendance de la justice est la pièce maîtresse de la transition démocratique ${ }^{19} »$. Or, de nombreux pays arabes font face à la corruption et à des décisions de justice plus politiques que juridiques, liées à une absence de culture démocratique, notamment chez les magistrats eux-mêmes. Ce fut l'une des revendications des citoyens lors des «printemps arabes». C'est dans ce contexte que certains États arabes ont tenté, ces dernières années, de réformer cette justice afin de reconquérir l'adhésion des citoyens, modifier leur regard sur cette institution et garantir le principe d'égalité devant la loi. Les constituants semblent être allés dans trois grandes directions afin de rénover le statut de la justice.

Une première initiative a consisté à travailler sur la question de l'indépendance des magistrats et la protection de celle-ci. C'est ainsi, par exemple, que la réforme constitutionnelle algérienne de février 2016 a complété l'article 148 de la loi fondamentale en disposant qu' « est proscrite toute intervention dans le cours de la Justice». "Le juge doit

19. Daniel Ludet et al., "La justice, un pouvoir de la démocratie », Terra nova, rapport, $1^{\mathrm{er}}$ mars 2011. 
se prémunir de toute attitude susceptible de porter atteinte à son impartialité. Le juge du siège est inamovible dans les conditions fixées par le statut de la Magistrature. » La constitution tunisienne de 2014 va dans le même sens. Les constitutions révolutionnaires égyptiennes mentionnent, quant à elles, que les juges sont «indépendants et inamovibles». La Constitution de 2014 vient même poser le principe selon lequel les juges peuvent être détachés, mais sous réserve des dispositions ayant pour objectif de "préserver l'indépendance du pouvoir judiciaire et l'impartialité des juges et de prévenir les conflits d'intérêts». Au Maroc, la révision de 2011 pose le principe selon lequel les magistrats doivent être nommés, dorénavant sur avis «conforme» du Conseil supérieur de la magistrature et non plus sur une simple "proposition » de celui-ci. Ainsi, le Conseil supérieur de la magistrature a depuis la possibilité de s'opposer à toute nomination à travers un avis qui lie le pouvoir exécutif.

Une deuxième direction semble être suivie afin d'assurer la rénovation de l'autorité judiciaire. Il s'agit d'assurer la mise en œuvre de l'indépendance de la justice en tant qu'institution. Ainsi, la révision constitutionnelle algérienne a posé le principe selon lequel le Conseil supérieur de la magistrature dispose de l'autonomie administrative et financière. La réforme marocaine (art. 113), elle, reconnaît de manière appuyée les missions du Conseil supérieur du pouvoir judiciaire. Il est indiqué que celui-ci veille à l'application des garanties accordées aux magistrats notamment quant à leur indépendance, leur nomination, leur avancement, leur mise à la retraite et leur discipline. Le Maroc est allé encore plus loin en modifiant les modalités de désignation des membres du conseil, mettant l'accent sur l'importance des membres élus tout en faisant membre de droit le médiateur et le président du Conseil des droits de l'homme. La présence de ce dernier montre bien la volonté du constituant marocain de placer le respect des droits de l'homme au centre du fonctionnement de la justice.

Enfin, les constituants arabes, afin d'entamer cette rénovation, ont, semble-t-il, chercher à consolider l'autorité de ce pouvoir. Cette idée se manifeste à travers plusieurs dispositions constitutionnelles. Il en a été ainsi lors de la révision constitutionnelle algérienne où l'on peut lire que «toute entrave à l'exécution d'une décision de justice est punie par la loi ». La constitution tunisienne reprendra cette idée en soulignant que les entraves aux décisions de justice " sans motifs légales sont interdites" (art. 111). La révision constitutionnelle marocaine, prenant acte de la pratique de certains juges consistant à satisfaire des intérêts privés portant atteinte à l'autorité de la justice, précise que tout manquement de 
la part du juge à ses devoirs d'indépendance et d'impartialité, constitue « une faute professionnelle grave».

\section{Une démocratisation constitutionnelle limitée}

Si l'on constate une certaine avancée dans la mise en place de principes démocratiques, il ne faut pas occulter les limites qu'il reste encore à supprimer afin de tendre vers une réelle démocratisation. Les déclarations politiques des uns et des autres ne peuvent minorer cette réalité qu'il convient d'infléchir ${ }^{20}$. En effet, certains droits fondamentaux restent encore à conquérir (3.1), le pouvoir exécutif demeure généralement prépondérant (3.2) et l'indépendance de la justice apparaît être toujours un enjeu (3.3).

\subsection{Des droits fondamentaux à conquérir}

Malgré les progrès réalisés, il n'en reste pas moins que les droits fondamentaux au sein du monde arabe restent à conquérir.

On peut constater, dans un premier temps, que les droits reconnus sont quelquefois limités dans leur formulation. C'est ainsi que la constitution syrienne interdit à tout groupement politique de se constituer sur une base religieuse ou professionnelle (art. 8). La liberté syndicale est réduite dans certains pays, comme au Maroc, au niveau de certaines professions dont les magistrats (art. 111). La constitution égyptienne interdit tout syndicat dans la fonction publique et limite chaque profession à un seul syndicat. De telles dispositions contreviennent à de nombreuses dispositions internationales. Une partie de ces dispositions, par leur manque de clarté ou de précision, peuvent servir de base à la restriction de certains droits. C'est ainsi que la révision constitutionnelle algérienne, en date de 2016, met l'accent sur la liberté de la presse. Néanmoins, il est mentionné que la diffusion des informations, des idées, des images et des opinions en toute liberté est garantie dans le cadre de la loi et, sous réserve, «du respect des constantes et des valeurs religieuses, morales et culturelle de la Nation». Les notions de «valeurs religieuses» ou de «valeurs morales et culturelles de la Nation» sont subjectives et laissées

20. «Nous œuvrons avec sérieux et détermination à l'édification d'un État de droit qui garantit les libertés individuelles et collectives, préserve les droits de l'Homme et promeut la pratique démocratique. À cet effet, j'invite les partis, les acteurs politiques et la société civile à contribuer à la diffusion de la culture et de la pratique démocratiques dans notre pays qui a déjà franchi de grandes étapes dans cette voie » (Discours du président mauritanien, Mohamed Ould Abdel Aziz à l'occasion du $52^{\mathrm{e}}$ anniversaire de l'indépendance nationale, 28 novembre 2012). 
à l'interprétation de chacun. Ne peut-on pas craindre une restriction de cette liberté de la presse par la loi, voire du juge a posteriori, au nom de certaines valeurs? Le flou de certaines notions est également présent au sein de la loi fondamentale du Bahreïn où, par exemple, les libertés d'opinion et d'expression sont garanties, mais à condition «que les croyances fondamentales de la doctrine islamique ne soient pas violées, l'unité du peuple compromise, la discorde ou le sectarisme semés ». Que faut-il entendre par "doctrine islamique», par «unité du peuple» ou encore par "discorde ou sectarisme » ? De même, la liberté d'association et la liberté syndicale sont garanties sous réserve que ces dernières soient fondées sur «des principes nationaux» et «[des] objectifs légitimes». Quelle signification donner aux notions de "principes nationaux» et d' "objectifs légitimes»? En outre, alors que l'égalité devant la loi est reconnue, on observe une certaine discrimination entre Algériens puisqu'on peut lire que «la nationalité algérienne exclusive est requise pour l'accès aux hautes responsabilités de l'État et aux fonctions politiques ». L'accès à certains emplois publics est verrouillé, comme la présidence suprême, puisqu'il faut «ne pas avoir acquis une nationalité étrangère; jouir uniquement de la nationalité algérienne d'origine et attester de la nationalité algérienne d'origine du père et de la mère ». On est également en droit de s'interroger sur la question des droits de la femme. Dans ce sens, par exemple, si la loi fondamentale du Bahreïn reconnaît l'égalité homme/femme, on remarque que l'accent est mis sur « les devoirs de la femme à l'égard de la famille ». Il est de plus ajouté que si une telle égalité est assurée, c'est sous réserve de ne pas «enfreindre» les dispositions de la «charia islamique». La Constitution du Bahreïn fait de l'héritage un droit "garanti et régi par la charia " et entraîne donc une certaine inégalité homme/femme en matière de droit de succession (art. 5). La référence à la charia peut, dans certains cas et notamment en ce qui concerne la femme, restreindre les droits. En effet, la charia s'éloigne des principes reconnus par les grands textes internationaux. Une telle situation peut surprendre dans la mesure où certains pays ont ratifié ces textes.

La conquête des droits fondamentaux est d'autant plus d'actualité que certaines constitutions restent très discrètes, voire silencieuses sur certains points. Il en est ainsi de la constitution syrienne, comme celle de l'Algérie, qui ne font aucunement référence aux grands textes internationaux touchant les droits de l'homme. Dans certains cas, les principes tel que celui de la non-rétroactivité de la loi pénale sont absents du texte fondamental. Il en va de même en Mauritanie ou encore en Syrie. La constitution mauritanienne reste, elle, très timide sur les droits 
sociaux essentiels tels que le droit de grève, le droit à la sécurité sociale, le droit à l'aide sociale ou encore le droit à un revenu d'existence pour toute personne qui se trouve dans l'incapacité de travailler ou de subvenir à ses besoins. Le droit à des moyens convenables d'existence est, lui aussi, absent de la constitution marocaine. Pourtant, il s'agit de droits fondamentaux et reconnus à ce titre par le droit international. Ils sont d'autant plus fondamentaux que l'on peut penser qu'ils conditionnent l'existence même des droits dits de "première génération" mettant la liberté au centre de ces derniers. En effet, peut-il y avoir une véritable liberté de la presse et d'opinion sans le droit à la reconnaissance d'un droit à l'éducation et à la culture? Une démocratie vivante peut-elle exister sans la reconnaissance de la liberté d'association, de réunion ou de la liberté syndicale ? Peut-il y avoir une véritable liberté du travail sans la reconnaissance de droits sociaux?

L'effectivité des droits fondamentaux semble également se heurter à leur mise en œuvre. Leur reconnaissance ne constitue pas une condition suffisante à leur réalité politique. Ainsi, malgré la reconnaissance par la constitution algérienne de l'égalité devant la loi et la reconnaissance du principe de non-discrimination, il est à noter qu'en matière d'actes civils ou commerciaux, là où le témoignage d'un homme suffit, il faut pour une femme deux témoignages. En matière d'héritage, une fille reçoit l'équivalent de la moitié de la part du garçon (il en est de même en Tunisie). Dans le même sens, la réforme constitutionnelle marocaine de 2011 fait du droit à la vie (art. 20) «le premier de tout être humain». Cependant, la peine de mort reste toujours applicable, même si elle n'est pas effective. La Tunisie témoigne également des limites de mise en œuvre des droits fondamentaux. On observe qu'en avril 2014 le gouvernement tunisien a levé les réserves formulées lors de la ratification de la Convention sur l'élimination de toutes les formes de discriminations à l'égard des femmes, ce qui apparaît, a priori, comme une bonne chose si ce n'est que la Tunisie a maintenu une déclaration générale indiquant qu'elle ne prendrait «aucune décision législative en conformité avec les exigences» de la convention si elles devaient se révéler incompatibles avec le chapitre 1 de la constitution tunisienne qui déclare que l'islam «est religion d'État». Au Maroc, la législation vient contredire les principes qui sont pourtant reconnus par la loi fondamentale dont celui de l'égalité homme/femme. En effet, par exemple, la mère ne peut accéder à la tutelle légale de ses enfants mineurs que sous certaines conditions très restrictives. En outre, alors que le Code de la famille souligne que la mère "aisée » a l'obligation de subvenir aux besoins de la famille en cas d'incapacité matérielle 
partielle ou totale du père (art. 199), cette responsabilité matérielle ne lui confère pas le droit à la tutelle légale sur ses enfants. De même, on est en présence d'une législation successorale inégalitaire qui participe à accroître la vulnérabilité des femmes vis-à-vis de la pauvreté. De plus, la pratique du habous et les règles régissant les terres collectives participent à déposséder les femmes de leurs droits à la terre et à la succession. Enfin, le Code de la nationalité reconnaît aux hommes le droit de transmettre leur nationalité à leur épouse étrangère alors que ce droit est dénié aux femmes.

Certaines peines sont disproportionnées par rapport aux faits incriminés. C'est ainsi que le Code pénal marocain, dans son article 491, prévoit une peine de prison d'un à deux ans pour adultère. La mise en œuvre des droits fondamentaux se heurte ainsi quelquefois à la jurisprudence des tribunaux. Le droit de grève a ainsi fait face à la jurisprudence des tribunaux, comme cela a été le cas en Égypte en 2015. Mettant en avant les intérêts des citoyens, la haute cour administrative égyptienne a validé une nouvelle législation interdisant aux fonctionnaires de décréter une grève sur leur lieu de travail. Selon la cour, cette mesure est conforme à la Constitution, étant donné qu'elle se fonde sur la charia ${ }^{21}$. Pourtant l'article 15 de la Constitution souligne clairement que la grève pacifique «est un droit réglementé par la loi». Au sein de certains pays la liberté d'expression est régulièrement remise en cause à travers des décisions de justice, malgré des dispositions constitutionnelles libérales. C'est ainsi qu'en mars 2014, en Égypte, trois adolescents coptes ont été condamnés par le tribunal correctionnel de Minya à cinq ans de prison en vertu de l'article 98 du Code pénal égyptien qui incrimine les atteintes à la religion. Ils ont été accusés "d'outrage à l'islam » pour avoir parodié, dans une vidéo, une scène de prière musulmane, en voulant se moquer des terroristes de Daech. Dans le même sens, Fatma Naout, écrivain reconnu, a été condamnée en mars 2016, pour «mépris de l'islam» à trois ans de prison parce qu'elle avait dénoncé, sur sa page Facebook, la cruauté représentée par l'égorgement en public d'animaux lors de la fête du sacrifice, l'Aïd al-Adha, qualifiant cette façon de faire de "vraie boucherie». Au Maroc, l'article 179 du Code pénal puni «d'un d'emprisonnement de 1 à 5 ans et d'une amende de 200 à 1000 dirhams toute offense commise envers la personne du roi ou de l'Héritier du trône; d'un emprisonnement de 6 mois à 2 ans et d'une amende de 200

21. Selon les explications avancées par la cour, la charia refuse toutes grèves portant des revendications dont l'importance reste en deçà des conséquences désastreuses subies par le pays et les citoyens. 
à 500 dirhams toute offense commise envers les membres de la famille royale». Les tribunaux font une application stricte et condamnent généralement aux peines les plus sévères les mis en cause. Cette doctrine jurisprudentielle réduit la portée de la liberté d'expression. C'est ainsi que le 13 février 2012 Abdessamad Adour, un jeune étudiant, a été condamné par le tribunal de première instance de Taza à trois ans de prison ferme pour «insultes à la personne du roi». Il avait critiqué le système politique et économique du Maroc lors de manifestations dans le cadre des "printemps arabes». De même, alors que la liberté de religion est constitutionnellement garantie dans la plupart des pays arabes, la législation elle-même vient contredire la loi fondamentale. C'est ainsi que l'article 222 du Code pénal marocain prévoit que «celui qui, notoirement connu pour son appartenance à la religion musulmane, rompt ostensiblement le jeûne dans un lieu public pendant le temps du Ramadan, sans motif admis par cette religion, est puni de l'emprisonnement d'1 à 6 mois ». Sur la base de cet article on a pu constater qu'en juin 2016 plusieurs Marocains, qui avaient rompu le jeûne, ont fait l'objet de poursuites ${ }^{22}$. Pourtant, la rupture du jeûne était justifiée par de fortes chaleurs. Un dernier exemple peut illustrer l'importance de la jurisprudence sur la portée de certains droits fondamentaux. Celui-ci concerne les droits de l'enfant. En effet, au Maroc l'âge légal pour se marier est fixé à 18 ans et il ne peut être déroger à cette règle que sous certaines conditions très strictes ${ }^{23}$. Cependant, on constate que, dans la pratique, des décisions judiciaires de plus en plus nombreuses octroient des dérogations à de jeunes filles mineures ${ }^{24}$.

Une autre critique peut être avancée et concerne l'absence ou l'insuffisance de mécanismes de protection des droits fondamentaux. Il aurait été opportun d'accorder une place beaucoup plus importante au juge comme garant des libertés individuelles. Il aurait été ainsi judicieux, pour

22. Il s'agissait de 2 porteurs de marchandises interpellés par la police pour avoir bu de l'eau pendant le ramadan. Ils se désaltéraient alors que la ville de Zagora connaissait de fortes chaleurs depuis plusieurs jours.

23. Extrait du Code de la famille, art. 19: «La capacité matrimoniale s'acquiert, pour le garçon et la fille, jouissant de leurs facultés mentales, à dix-huit années grégoriennes révolues »; art. 20 : «Le juge de la famille chargé du mariage peut autoriser le mariage du garçon et de la fille avant l'âge de la capacité prévu à l'article 19 ci-dessus, par décision motivée précisant l'intérêt et les motifs justifiant ce mariage, après avoir entendu les parents du mineur ou son représentant légal, et après avoir eu recours à une expertise médicale ou procédé à une enquête sociale. La décision du juge autorisant le mariage d'un mineur n'est susceptible d'aucun recours. »

24. Mariam Monjid, «Le mariage du mineur en droit marocain», RIDC 2015, p. 207. 
la révision constitutionnelle algérienne, de préciser que seule une décision de justice peut dissoudre une association ou encore, pour la constitution révolutionnaire égyptienne, de mentionner que la fermeture d'un journal, par exemple, ne peut intervenir que sur la base d'une décision judiciaire. Au Bahreïn, comme en Mauritanie, nous remarquons l'absence de contrôle de constitutionnalité par voie d'exception, alors que celui-ci constitue une garantie supplémentaire reconnue. De même, on observe que lorsqu'un contrôle constitutionnel existe encore faut-il qu'il soit effectif. C'est ainsi qu'au Maroc de mars 1994 à mars 2009 le Conseil constitutionnel a examiné la constitutionnalité de seulement 10 lois ordinaires et les saisines n'étaient pas forcément fondées sur la violation de libertés publiques ou droits fondamentaux ${ }^{25}$. Il y a ainsi, comme le souligne le professeur Bernoussi, une réelle timidité du Conseil constitutionnel à faire référence au préambule de la Constitution dans ses décisions ${ }^{26}$. Il existe une certaine peur de porter atteinte à la souveraineté nationale. Ce manque de courage peut s'expliquer par le manque de culture démocratique des membres du Conseil constitutionnel, mais également du fait que certaines législations soient impulsées par le monarque lui-même. Il ne faut pas oublier que le roi lui-même est amené à nommer certains membres du conseil. C'est pourquoi il serait nécessaire de consolider l'indépendance des membres de ces cours.

L'indépendance juridique ne signifie pas l'absence de dépendance politique. La solution serait, peut-être comme en Bolivie, de faire en sorte que les membres des cours constitutionnelles puissent être élus. Afin d'accroître l'efficacité de ces cours, il serait bon de reconnaître explicitement leur compétence afin qu'elles puissent contrôler la conformité des lois constitutionnelles elles-mêmes aux conventions internationales ratifiées par un pays. Il s'agirait là d'une procédure préventive qui irait dans le sens d'une meilleure protection de l'État de droit ${ }^{27}$.

Enfin, les droits fondamentaux restent d'autant plus à conquérir dans le monde arabe que, même si certains d'entre eux bénéficient d'une véritable reconnaissance, ils font l'objet dans le même temps de nombreuses violations. C'est ainsi qu'en ce qui concerne la liberté de la presse, selon le classement de Reporters sans frontières de 2015, le Maroc se situe dans

25. «L'autorité des décisions du Conseil constitutionnel/Maroc», Nouveaux cahiers $d u$ Conseil constitutionnel, 2011.

26. Nadia Bernoussi et Abdelrrahim Maglouhi, art. cité, p. 479.

27. Philippe Blacher, «Le contrôle par le Conseil constitutionnel des lois constitutionnelles », RDP 2016, p. 545. 
la zone rouge, au $131^{\mathrm{e}}$ rang mondial, loin derrière la Mauritanie et la Tunisie $^{28}$. Dans la Tunisie issue du «printemps» de 2011, les homosexuels courent toujours le risque d'être envoyés derrière les barreaux. C'est ainsi que le 22 septembre 2015 un jeune Tunisien a été condamné à 1 an de prison par le tribunal de première instance de Sousse en application de l'article 230 du Code pénal qui réprime l'homosexualité. En Mauritanie, malgré la réforme constitutionnelle de 2012 allant dans le bon sens, on note que le 2 janvier 2014 un homme de 28 ans, comptable, avait été interpellé pour avoir été le premier à avoir critiqué les décisions prises par le prophète et, le 24 décembre 2014, la Cour criminelle de Nouadhibou l'a condamné à mort pour blasphème.

28. Les médias imprimés et en ligne indépendants continuent d'enquêter et de critiquer les membres et les politiques du gouvernement, mais sont confrontés à des poursuites et au harcèlement dès qu'ils franchissent certaines lignes. Le Code de la presse prévoit des peines de prison pour diffusion «de mauvaise foi » d'une «nouvelle fausse » susceptible, selon les autorités, de troubler l'ordre public, ou pour des propos jugés diffamatoires. La télévision d'État marocaine offre une certaine marge pour le débat et le journalisme d'investigation, mais peu de latitude pour la critique directe du gouvernement ou pour les points de vue éloignés des positions officielles sur les questions clés. Les autorités ont continué leur enquête sur des accusations de terrorisme à l'encontre d'Ali Anouzla, directeur du site d'information indépendant Lakome.com, en raison d'un article décrivant et fournissant un lien indirect vers une vidéo militante islamiste de recrutement. En 2013, Anouzla a passé 5 semaines en détention après avoir publié cet article. Le rappeur Mouad Belghouat, connu sous le nom d' "Al-Haqed" (L'indigné), dont les chansons dénoncent la corruption et les abus commis par la police, a passé 4 mois en prison après avoir été condamné pour des agressions contre des policiers, lors d'un procès où le juge $\mathrm{a}$ refusé de convoquer des témoins à décharge ou des victimes présumées. En février, les autorités ont empêché une librairie de Casablanca d'accueillir un événement à l'occasion du nouvel album d'Al-Haqed. Othmane Atiq, un rappeur âgé de 17 ans connu sous le nom de "Mister Crazy", a purgé une peine de 3 mois de prison pour insultes à la police et incitation à la consommation de drogue à cause de ses clips dépeignant la vie de la jeunesse urbaine marginalisée. Abdessamad Haydour, un étudiant, a purgé une peine de 3 ans de prison pour avoir traité le roi de «chien », de "meurtrier» et de " dictateur» dans une vidéo diffusée sur YouTube. Un tribunal l'a condamné en février 2012 en vertu d'une disposition du Code pénal criminalisant toute "offense commise envers la personne du roi » (Human rights watch, Rapport mondial 2015. Pertinence des droits humains dans les moments difficiles, http://http ://www.hrw.org/fr/news/2015/01/29/rapport-mondial2015-pertinence-des-droits-humains-dans-les-moments-difficiles. 


\subsection{La domination du pouvoir exécutif}

Malgré des avancées, les régimes politiques arabes restent imprégnés par un pouvoir exécutif fort. En effet, d'une part le gouvernement maintient son autorité (3.2.1), le chef de l'État bénéficie encore d'une certaine suprématie (3.2.2) et nous constatons une pratique du pouvoir au bénéfice du pouvoir exécutif (3.2.3).

\subsubsection{Le maintien de l'autorité du gouvernement}

Le gouvernement au sein du monde arabe conserve une autorité certaine. Cette autorité trouve son fondement dans la loi fondamentale elle-même. Dans le gouvernement, il faut remarquer que Premier ministre lui-même dispose d'un rôle prépondérant. C'est ainsi qu'au Maroc le chef du gouvernement peut dissoudre la chambre des représentants (art. 104). De même, il peut engager la responsabilité du gouvernement devant la chambre des représentants, sur une déclaration de politique générale ou sur le vote d'un texte, et la confiance ne peut lui être refusée qu'à la majorité absolue des membres de celle-ci (art. 103). Au sein de la constitution algérienne, on peut constater que le gouvernement, et plus particulièrement le Premier ministre, possède une place prépondérante dans la procédure législative. En effet, en cas de désaccord entre les deux chambres, le Premier ministre a la possibilité de demander la réunion, dans un délai maximal de 15 jours, d'une commission paritaire constituée de membres des deux chambres pour proposer un texte sur les dispositions objet du désaccord. On note également que le Premier ministre possède l'initiative des lois (art. 136). À côté de la place particulière du chef du gouvernement, les différentes constitutions arabes tendant à préserver le statut protecteur du gouvernement dans son ensemble. Cette autorité du gouvernement se matérialise dans la procédure législative, comme en témoigne la constitution mauritanienne. C'est ainsi que le gouvernement possède, là aussi, l'initiative des lois. En outre, après l'ouverture du débat, il peut s'opposer à l'examen de tout amendement qui n'a pas été antérieurement soumis à la commission et, si le gouvernement le demande, l'assemblée saisie se prononce par un seul vote sur tout ou partie du texte en discussion en ne retenant que les amendements proposés ou acceptés par lui (art. 65). On constate également que le gouvernement a la maîtrise de l'ordre du jour puisqu'il comporte, par priorité et dans l'ordre que le gouvernement a fixé, la discussion des projets et des propositions de loi acceptés par lui (art. 69). 


\subsubsection{La prééminence du chef de l'État}

Mais encore plus que le gouvernement qui est l'élément mobile de l'exécutif, l'élément fixe constitué par le chef de l'État (président de la République ou roi) possède une place prépondérante dans l'architecture de ces constitutions. C'est ainsi que dans la constitution mauritanienne, le gouvernement assure seulement «la mise en œuvre de la politique générale de l'État conformément aux orientations et aux options fixées par le président de la République» (art. 43) qui « détermine et conduit la politique extérieure de la Nation ainsi que sa politique de défense et de sécurité ${ }^{29}$ "(art. 30). Le gouvernement se trouve ainsi sous la tutelle directe du chef de l'État. En Mauritanie, le Premier ministre et l'ensemble des ministres «sont responsables devant le Président de la République $^{30} »$ (art. 30). La constitution égyptienne fait du gouvernement un simple « collaborateur» du président de la République (art. 167) et la Constitution du Bahreïn est encore plus explicite puisque «le roi exerce ses pouvoirs directement ou par l'intermédiaire de ses ministres » (art. 33). En Syrie, il revient au chef de l'État de nommer tant le chef du gouvernement que ses membres et il a compétence pour les « décharger de leurs fonctions» (art. 97). En Égypte, le président de la République peut même dispenser le gouvernement de mener à bien ses tâches s'il obtient l'approbation de la majorité de la chambre des représentants (art. 147). En Tunisie, le chef de l'État possède de véritables moyens de pression sur le gouvernement, car il a la possibilité de demander à l'Assemblée des représentants du peuple de procéder à un vote de confiance à l'encontre du gouvernement (art. 99). Au Maroc, en janvier 2015, le ministre de la Jeunesse et des Sports, Mohamed Ouzzine fut démis de ses fonctions à l'initiative du roi ${ }^{31}$.

29. Il en sera de même au sein du nouveau texte constitutionnel de la Tunisie dans lequel « le président de la République représente l’État ». «Il détermine les politiques générales dans les domaines de la défense, des relations étrangères et de la sécurité nationale relative à la protection de l'État et du territoire national contre toute menace intérieure ou extérieure, après consultation du chef du Gouvernement » (art. 77).

30. Il en est de même en Syrie (art. 121 de la constitution).

31. La décision est intervenue après un rapport présenté au souverain chérifien sur les dysfonctionnements qu'a connus l'un des matches de la Coupe du monde des clubs. Mohamed Ouzzine avait d'abord présenté une lettre de démission au Premier ministre, Abdelilah Benkirane, qui l'avait transmise à Mohammed VI. Le roi a décidé de limoger M. Ouzzine «pour ses manquements dans la rénovation du stade de Rabat». 
L'influence du chef de l'État se manifeste également sur le pouvoir législatif. Par exemple, il peut prendre la parole au sein des assemblées afin de défendre sa politique et donner de véritables consignes de vote aux parlementaires. C'est le cas en Syrie par exemple (art. 101). Il possède également un véritable moyen de pression au travers du droit de dissolution, ce qui lui permet d'assurer la discipline politique au sein des chambres. Il en est ainsi en Syrie (art. 11), au Maroc (art. 96) ou encore au Bahreïn où le roi n'a pas à consulter qui que ce soit. Le chef de l'État est également à l'initiative des lois, ce qui lui permet de proposer ses propres textes à sa majorité dans le cas d'un contexte de «fait majoritaire ». Tel est le cas en Syrie (art. 112) ou encore en Tunisie (art. 32). On constate qu'il possède aussi un pouvoir de veto qui, même s'il n'est que partiel, n'en reste pas moins important puisqu'il peut s'opposer à la promulgation d'une loi qui devra alors recueillir un vote à la majorité qualifiée afin de contraindre le chef de l'État à la promulguer. Cette majorité qualifiée, difficile à atteindre, joue en faveur du chef de l'État. En Syrie ainsi qu'en Algérie, le nouveau vote devra ainsi se faire à la majorité des deux tiers, alors qu'en Tunisie la majorité absolue des membres composant l'assemblée est demandée. Au Maroc, le roi reste très présent dans ses rapports avec le pouvoir législatif (droit d'initiative relative à la révision constitutionnelle, prise de parole devant le parlement...). Cette emprise $\mathrm{du}$ roi a pu faire dire au professeur Duguit qu'on ne peut qualifier une monarchie de "modérée» ou encore de «limitée » lorsqu'il n'y a pas de véritable partage du pouvoir entre les deux grandes institutions constitutionnelles ${ }^{32}$.

On observe également que les chefs d'État arabes tendent à posséder un rôle clé dans le domaine de la procédure touchant la révision de la Constitution, voire un pouvoir de veto. C'est le cas en Algérie où on remarque que si les trois quarts des membres des deux chambres réunies du parlement peuvent proposer une révision constitutionnelle, il revient au chef de l'État de juger de l'opportunité de soumettre ou pas le texte au référendum. En Syrie, une proposition de révision, votée par l'Assemblée populaire, ne pourra être validée définitivement que sous réserve de «son approbation par le Chef de l'État» (art. 150). En outre, on constate que certaines constitutions protègent le chef de l'État en empêchant toute modification de son statut. Il en est ainsi de l'article 175 de la constitution marocaine qui pose le principe selon lequel «aucune révision ne peut porter [...] sur la forme monarchique de l'État ». De même l'article 120

32. Léon Duguit, Traité de droit constitutionnel, Paris, De Boccard, t. 2, 1928, p. 773. 
de la Constitution du Bahreïn met en avant le fait que «l'existence de la monarchie constitutionnelle et le principe de la succession héréditaire au Bahreïn [...] ne peuvent faire l'objet d'une proposition de révision ».

Enfin, on remarque que ces mouvements libéraux constitutionnels n'ont pas tempéré les pouvoirs exorbitants en temps de crise. Le danger n'est pas dans le fait d'être titulaire de tels pouvoirs qui peuvent être justifiés dans des situations de crise extrêmes, mais dans le peu d'encadrement dont ces pouvoirs exceptionnels font l'objet contrairement aux démocraties occidentales (art. 16 en France, art. 138 au Portugal). La Constitution du Maroc illustre ces limites. C'est ainsi que l'article 59 dispose que « lorsque l'intégrité du territoire national est menacée ou que se produisent des événements qui entravent le fonctionnement régulier des institutions constitutionnelles, le roi peut [...] proclamer par dahir l'état d'exception ». "De ce fait, le roi est habilité à prendre les mesures qu'imposent la défense de l'intégrité territoriale et le retour, dans un moindre délai, au fonctionnement normal des institutions constitutionnelles. » On remarque bien que ce texte ne permet pas d'encadrer le pouvoir du souverain. Aucun organe ne vient contrôler la mise en œuvre des dahirs adoptés. Le parlement n'a pas à se prononcer sur les mesures prises dans le cadre de l'état d'exception. Le parlement, même s'il ne peut pas être dissous, se pose néanmoins la question suivante: doit-il siéger obligatoirement? D’autre part, il n'est fait aucune référence au gouvernement, au rôle et à la place de celui-ci pendant cette période de crise. Le Conseil constitutionnel, qui est garant des libertés et des droits fondamentaux, semble également complètement écarté de la procédure. La loi fondamentale de la Syrie est encore plus sommaire sur l'encadrement de ces pouvoirs exceptionnels en temps de crise. En effet, l'article 114 de la Constitution dispose simplement que le président de la République, si un grand danger menace l'unité nationale, la sécurité ou l'indépendance de la patrie ou empêche les institutions de l'État de remplir leurs obligations constitutionnelles, peut prendre les mesures d'urgence exigées par les circonstances, en vue de faire disparaître ce danger. On observe qu'aucun contrôle n'est organisé. En Algérie, le président de la République a seul le pouvoir de déclarer l'état d'exception, d'en déterminer l'opportunité et la mise en œuvre même s'il doit consulter le président du Conseil de la nation, le président de l'Assemblée populaire nationale et le président du Conseil constitutionnel. Mais il s'agit là d'une simple consultation qui ne saurait le lier, d'autant que la configuration politique de l'Algérie réduit la portée de ces consultations. La Constitution lui donne toute compétence afin de prendre les mesures exceptionnelles qui s'imposent et assurer la protection des institutions. La loi fondamentale ne donne 
aucune indication sur les modalités de contrôle des mesures prises, ni sur leur nature.

\subsubsection{Une pratique du pouvoir au service de l'exécutif}

La pratique du pouvoir constitue un élément, une variable fondamentale permettant d'illustrer l'emprise de l'exécutif sur le fonctionnement des institutions. Comme le soulignait très bien le doyen Duguit: «[L]e droit n'est point cette construction édifiée de toutes pièces par les juristes sur le fondement peu stable du droit individuel ou de l'omnipotence de l'État, $[. .$.$] tout cet ensemble de fictions et d'abstractions s'évanouit$ à la simple observation de la réalité ${ }^{33}$. » Olivier Beaud précise que «ceux qui nient soit l'élément juridique, soit l'élément politique dans la constitution, ratent la spécificité d'une notion qui ne se saisit qu'à partir de la charnière du droit et du politique ${ }^{34}$ ».

Les pratiques de certains chefs d'État arabes témoignent de leur forte autorité au sein des institutions. C'est ainsi qu'en ce qui concerne la révision constitutionnelle marocaine, c'est le roi lui-même qui a nommé les membres de la commission en charge de rédiger le projet constitutionnel qui sera soumis au peuple. De même, lorsqu'on observe les modalités de la révision constitutionnelle de février 2016 en Algérie, on constate que c'est le président Bouteflika qui a désigné les membres de la commission chargée de proposer le projet de révision constitutionnelle. Il en ressort que les membres des commissions de révision, s'ils peuvent délibérer librement doivent le faire sous réserve de respecter le cadre fixé par le chef de l'État. En outre au Maroc il fut impossible de débattre sur la question du statut de la royauté ou encore de la place de la religion.

On remarque que les têtes «mobiles» des exécutifs arabes, c'est-à-dire les gouvernements, restent souvent sous le contrôle du chef de l'État. Il en est ainsi au Maroc où le Premier ministre lui-même a pu déclarer en novembre 2011 que «le roi est le chef de l'État et aucune décision importante ne peut être prise en Conseil des ministres sans la volonté du roi ». En novembre 2013, il ajoutera: «Le Maroc ne connait pas de cohabitation à la française; chez nous, la constitution précise que le roi est le chef de l'État. Il est donc mon supérieur hiérarchique, mon patron. » Il n'est pas rare que le souverain reçoive certains membres du

33. Ibid.

34. Olivier Beaud, «L'histoire du concept de constitution en France. De la constitution politique à la constitution comme statut juridique de l'État », Jus Politicum, $\mathrm{n}^{\circ} 3$, p. 2-3 et 29 . 
gouvernement ou le chef du gouvernement lui-même afin de leur faire part de son point de vue et donner certaines directives sur une question particulière. Ainsi, en janvier 2016, le souverain a pu recevoir le Premier ministre et lui donner ses instructions concernant l'approvisionnement en eau potable des "villages éloignés et excentrés». Au Bahreïn, le Premier ministre, qui gouverne le pays, n'est autre que cheikh Khalifa ben Salmane Al Khalifa qui est l'oncle du roi Hamad ben Issa Al Khalifa, ce qui le fait apparaître comme un simple exécutant des décisions royales. En septembre 2015, le président égyptien Abdel Fattah al-Sissi a demandé la démission du gouvernement et a chargé Chérif Ismail, ministre du Pétrole dans le gouvernement démissionnaire, de former un nouveau cabinet.

Dans le domaine législatif, on retrouve une certaine emprise du chef de l'État sur le parlement. C'est ainsi que dans son discours devant le nouveau parlement élu le 13 avril 2013 le président Bachar el-Assad n'hésite pas à mettre en avant la politique à suivre dans le domaine économique, au niveau international comme national ${ }^{35}$. Il s'agit de véritables directives données au pouvoir législatif. En outre, il ne faut pas oublier que c'est le parti Baas qui détient la majorité absolue des sièges à l'Assemblée du peuple. Cela assure au chef de l'État une certaine autorité tant sur la majorité parlementaire que sur le gouvernement et le Premier ministre. L'emprise politique du chef de l'État sur le pouvoir législatif se matérialise explicitement à travers l'exemple algérien, où le chef de l'État, en cas d'état d'exception, peut légiférer par ordonnances (art. 142) et donc dessaisir le parlement de sa compétence ${ }^{36}$. Au Maroc, le souverain, à l'occasion de discours devant les deux chambres du parlement, n'hésite pas à adresser ses directives aux parlementaires. C'est ainsi que lors d'un discours le 9 octobre 2015, il a pu déclarer: «Nous avons jugé nécessaire de rappeler [...] au parlement la nécessité de se conformer aux dispositions de l'article 86 de la Constitution, qui fixe le terme de cette législature comme dernier délai pour soumettre les projets de lois organiques à l'approbation du parlement. Nous rappelons ici, à titre d'exemple, les projets des lois organiques relatives à la mise en œuvre du caractère officiel de la langue amazighe, au Conseil national des langues et de la culture marocaine, à l'exercice du droit de grève et au Conseil de régence. [...] Par ailleurs, l'élaboration du projet de loi organique sur

35. http://www.legrandsoir.info/discours-du-president-syrien-devant-le-nouveauparlement-elu-le-13-avril-2016-texte-integral.html.

36. Lorsque le pays est menacé d'un péril imminent dans ses institutions, dans son indépendance ou dans son intégrité territoriale. 
la grève nécessite d'engager de larges consultations et de faire preuve d'un esprit de consensus constructif, de façon à garantir les droits des travailleurs, les intérêts du patronat et l'intérêt de la Nation. »

\subsection{Le pouvoir judiciaire : une consolidation nécessaire}

Malgré certaines avancées dans le sens de l'État de droit au sein des constitutions post-révolutionnaires, il n'en reste pas moins que ces pays n'ont pas su aller jusqu'au bout du processus démocratique, notamment, dans le domaine de la justice. Les réformes constitutionnelles apparaissaient pourtant comme une opportunité offerte d'aller au-delà d'une application minimaliste des principes traversant la justice et internationalement reconnus tant par le droit international que par les constitutions occidentales.

La question fondamentale se rapportant au pouvoir judiciaire est son indépendance. Sans indépendance le pouvoir judiciaire n'est qu'un instrument au service du politique. Le droit serait dès lors régulièrement écarté, interprété dans le sens des faveurs du pouvoir ou de groupes de pressions économiques. Il s'en suivrait non seulement des ruptures d'égalité devant la loi, un certain arbitraire, mais également des atteintes aux libertés fondamentales. C'est pourquoi les instruments internationaux prêtent une attention particulière à l'indépendance de la justice. Indépendance qui est un des critères de tout régime qualifié de démocratique. Ce principe se retrouve ainsi dans la Déclaration universelle des droits de l'homme ${ }^{37}$, du Pacte international relatif aux droits civils et politiques ${ }^{38}$, de la Convention européenne de sauvegarde des droits de l'homme ${ }^{39}$, de la Charte des droits fondamentaux de

37. Art. 10: «Toute personne a droit, en pleine égalité, à ce que sa cause soit entendue équitablement et publiquement par un tribunal indépendant et impartial, qui décidera soit de ses droits et obligations, soit du bien-fondé de toute accusation en matière pénale dirigée contre elle.»

38. Art. 14: 1. «Tous sont égaux devant les tribunaux et les cours de justice. Toute personne a droit à ce que sa cause soit entendue équitablement et publiquement par un tribunal compétent, indépendant et impartial, établi par la loi, qui décidera soit du bien-fondé de toute accusation en matière pénale dirigée contre elle, soit des contestations sur ses droits et obligations de caractère civil.»

39. Art. 6. Droit à un procès équitable: «Toute personne a droit à ce que sa cause soit entendue équitablement, publiquement et dans un délai raisonnable, par un tribunal indépendant et impartial, établi par la loi, qui décidera soit des contestations sur ses droits et obligations de caractère civil, soit du bien-fondé de toute accusation en matière pénale dirigée contre elle.» 
l'Union européenne ${ }^{40}$, la Charte africaine des droits de l'homme et des peuples (art. 7) ou encore la Charte arabe des droits de l'homme (art. 12). De nombreuses recommandations d'instances internationales rappellent régulièrement l'importance de ce principe comme les Nations unies $^{41}$ et le Conseil de l'Europe ${ }^{42}$. Pourtant, on ne peut que regretter les limites du nouveau constitutionnalisme arabe. En effet, à titre d'exemple, la constitution mauritanienne, pourtant révisée en 2012, reste silencieuse sur le principe de l'inamovibilité des juges, sur l'interdiction de toute pression politique ou privée. Le principe de l'impartialité des juges n'est même pas posé. Pour préserver plus concrètement l'indépendance des magistrats, il aurait été opportun de faire interdiction aux magistrats d'exercer toute autre fonction publique, de s'adonner à toute activité lucrative, professionnelle ou salariée, et à toute activité politique. L'indépendance des magistrats passe aussi par une rémunération correcte destinée à éviter toute corruption. Au Maroc, s'il est fait référence aux notions d'indépendance et d'inamovibilité, cela concerne uniquement les magistrats du siège. Il faut en déduire que les magistrats du parquet ne sont pas indépendants. On constate dans ce sens que l'article 110 dispose expressément que «les magistrats du parquet sont tenus à l'application du droit et doivent se conformer aux instructions écrites émanant de l'autorité hiérarchique». On remarque également, qu'afin de garantir leur indépendance, les magistrats ne peuvent adhérer à des partis politiques ou à des organisations syndicales. Cependant, on peut aussi voir cette interdiction comme une limite à la liberté d'expression des magistrats. Il semble que le constituant ait peur que les magistrats

40. Arti. 47. Droit à un recours effectif et à accéder à un tribunal impartial: «Toute personne dont les droits et libertés garantis par le droit de l'Union ont été violés a droit à un recours effectif devant un tribunal dans le respect des conditions prévues au présent article. Toute personne a droit à ce que sa cause soit entendue équitablement, publiquement et dans un délai raisonnable par un tribunal indépendant et impartial, établi préalablement par la loi. Toute personne a la possibilité de se faire conseiller, défendre et représenter. Une aide juridictionnelle est accordée à ceux qui ne disposent pas de ressources suffisantes, dans la mesure où cette aide serait nécessaire pour assurer l'effectivité de l'accès à la justice. »

41. Voir notamment les principes fondamentaux relatifs à l'indépendance de la magistrature adoptés par le $7^{\mathrm{e}}$ congrès des Nations unies pour la prévention du crime et le traitement des délinquants qui s'est tenu à Milan du 26 août au 6 septembre 1985 et confirmés par l'assemblée générale dans ses résolutions 40/32 du 29 novembre 1985 et 40/146 du 13 décembre 1985, et les principes directeurs applicables au rôle des magistrats du parquet adoptés par le $8^{\mathrm{e}}$ congrès des Nations unies pour la prévention du crime et le traitement des délinquants qui s'est tenu à La Havane (Cuba) du 27 août au 7 septembre 1990.

42. Cons. Europe, 13 oct. 1994, recomm. N R (94) 12. 
puissent s'organiser et éventuellement contester des réformes législatives portant atteinte à leur statut, leur liberté et leur indépendance, voire faire état de leur inquiétude sur d'éventuelles législations liberticides. Il faut également noter que lorsque des instances dont l'objectif est, notamment, de garantir cette indépendance sont créées, leur portée doit, semble-t-il, être relativisée. Ainsi, au Maroc, c'est le Conseil supérieur du pouvoir judiciaire qui est garant de l'indépendance des magistrats. Or, on observe que ce conseil semble être sous le contrôle du palais. En effet, parmi les 30 membres nous trouvons: le premier président de la Cour de cassation en qualité de président délégué, le procureur général du roi près la Cour de cassation, le président de la première chambre de la Cour de cassation, le médiateur, le président du Conseil national des droits de l'homme, 5 personnalités qualifiées. L'ensemble de ces personnes est nommé directement ou indirectement par le roi. Le Conseil supérieur du pouvoir judiciaire peut être d'autant plus critiqué sur son indépendance que l'article 11 de la Constitution dispose que «dans les affaires concernant les magistrats du parquet, le Conseil supérieur du pouvoir judiciaire prend en considération les rapports d'évaluation établis par l'autorité hiérarchique dont ils relèvent ».

Enfin, différents exemples au sein de l'histoire politique des pays arabes peuvent venir illustrer la dépendance du pouvoir judiciaire à l'égard du pouvoir exécutif. C'est ainsi que le président Abdelaziz Bouteflika, dans un décret présidentiel du 28 février 2016, a officiellement mis fin aux fonctions du procureur général Belkacem Zeghmati près de la Cour d'Alger. De même en Égypte, en 2012, d'influents juges ont apporté leur soutien au procureur général, qui refusait de quitter son poste après avoir été limogé par le président Mohamed Morsi. La même année la présidence mauritanienne annonçait à Seyid ould Ghaylani qu'elle mettait fin à son mandat de président de la Cour suprême. Il s'agissait d'un véritable limogeage. En effet, la loi précise que le président de la Cour suprême ne peut être suspendu ou démis de ses fonctions que dans un nombre de cas précis et restreints: à sa demande, pour cause d'incapacité physique, en cas de perte des droits civiques et politiques, ou en cas de manquement à l'honneur et à la dignité de sa fonction. Or le mandat de Seyid ould Ghaylani, nommé par décret en 2010, courrait jusqu'en 2015 et le magistrat affirme avoir immédiatement informé la présidence de son refus de quitter son poste. Certains réflexes judiciaires sont encore présents. C'est ainsi qu'en 2003, le tribunal administratif de Rabat avait annulé le refus du directeur de la Sûreté nationale de recruter, comme officier de police, un candidat qui se prévalait d'une recommandation de son altesse royal le prince héritier pour obtenir le 
poste sans passer le concours (la chambre administrative de la Cour suprême infirmera cependant ce jugement ${ }^{43}$ ). On voit bien ici comment l'autorité judiciaire est traversée par des réflexes qui sont bien loin de la légalité.

\section{Conclusion}

On constate ainsi que des mouvements constitutionnels sont à l'œuvre dans le monde arabe. De telles mutations, même si elles peuvent paraître limitées, s'intègrent cependant dans un processus de démocratisation qui répond aux attentes des peuples arabes aujourd'hui. Il reste néanmoins que ce processus, par sa définition même, est inabouti, tout en se situant dans une certaine dynamique. Plusieurs évolutions restent à venir : la consolidation des droits fondamentaux; une autre pratique du pouvoir pour un meilleur équilibre entre les pouvoirs. Mais plus encore, il reste aux peuples arabes à s'approprier ce processus. De manière générale, comme le soulignait Pierre Mendés France, «la démocratie est d'abord un état d'esprit ${ }^{44} »$. N'est-ce pas, peut-être, sur cette question, que le monde arabe doit aussi avancer?

Maître de conférences en droit public Université Toulouse-Jean Jaurès (Centre de recherches et d'études sur les droits fondamentaux, CREDOF, Paris X)

43. Nadia Bernoussi et Abdelrrahim Maglouhi, art. cité, p. 479.

44. La République moderne, Paris, Gallimard, 1962. 